\title{
Desarrollo Profesional del Docente de Matemáticas a través de sus Tareas para el Aula propuestas en un Curso de Formación"
}

\author{
Professional Development of Mathematics Teacher from their Classroom \\ Tasks proposed in a Teacher Education Course
}

\author{
Elisabeth Ramos-Rodríguez ${ }^{* *}$ \\ Pablo Flores Martínez ${ }^{* * *}$ \\ João Pedro da Ponte ${ }^{* * * *}$ \\ Antonio Moreno Verdejo ${ }^{* * * * *}$
}

\begin{abstract}
Resumen
Parte de un trabajo doctoral, enmarcado dentro de la línea de formación de profesores, es analizar las tareas de enseñanza propuestas por docentes en un curso de formación realizado en Chile, en el año 2012, en donde sus participantes tienen que identificar un problema profesional referido a la enseñanza del álgebra y profundizar sobre el mismo diseñando, implementando y analizando tareas matemáticas para una clase. Luego de presentar algunos descriptores de las tareas, con una metodología cualitativa empleamos el análisis de contenido para describir la evolución de las tareas de enseñanza propuestas por dos profesoras participantes de este curso formativo. El análisis de los datos ponen de manifiesto cómo las docentes afrontan un problema de su práctica, incorporando nuevos elementos de actuación respecto al diseño de tareas, incrementando en sus conocimientos, en específico, en relación con la noción de modelación, avanzando en este proceso en su desarrollo profesional.
\end{abstract}

Palabras clave: Formación de Profesores. Tareas Matemáticas. Problemas del Álgebra.

\footnotetext{
"Este estudio ha sido financiado por la Pontificia Universidad Católica de Valparaíso (Chile) y por medio de una Beca del gobierno de Chile a través de la Comisión Nacional de Investigación Científica y Tecnológica, CONICYT. Además, forma parte del proyecto de investigación EDU2012-33030, Procesos de aprendizaje del profesor de matemáticas en formación, de la Dirección General de Investigación Científica y Técnica del Ministerio de Comercio e Innovación de España.

** Doctoranda del programa de posgrado de Ciencias de la Educación de la Universidad de Granada (UGR). Profesora del Instituto de Matemáticas de la Pontificia Universidad Católica de Valparaíso (PUCV), Valparaíso, Chile. Dirección postal: Avenida Brasil, 2950, Valparaíso, Chile. E-mail: elisabeth.ramos@ucv.cl

**** Doctor en Matemáticas por la Universidad de Granada. Profesor Titular de la Universidad de Granada (UGR), Granada, España. Dirección postal: La Cartuja, s/n, 18011, Granada, España. E-mail: pflores@ugr.es

***** Doutor em Educação Matemática pela Universidade da Georgia, EUA. Professor Catedrático no Instituto de Educação da Universidade de Lisboa (IE ULisboa), Lisboa, Portugal. Endereço: Alameda da Universidade, 1649-013, Lisboa, Portugal. E-mail:jpponte@ie.ulisboa.pt.

****** Doctor en Didáctica de las Matemáticas por la Universidad de Granada. Profesor de la Universidad de Granada (UGR), Granada España. Dirección postal: La Cartuja, s/n, 18011, Granada, España. E-mail: amverdejo@ugr.es.
} 


\begin{abstract}
Part of a doctoral work, framed within the teacher training online, is to analyze the tasks proposed by teachers teaching in a training course held in Chile in 2012, where participants have to identify a professional problem referred to teaching algebra and deepen on it designing, implementing and analyzing mathematical tasks for a class. After presenting some descriptors of tasks, with a qualitative methodology we use content analysis to describe the evolution of teaching tasks proposed by two teachers participating in this training course. Analysis of the data show how teachers face a problem of practice, incorporating new elements of action regarding the design of tasks, increasing their knowledge, specifically in relation to the notion of modeling, through this process in their professional development.
\end{abstract}

Keywords: Teacher Education. Math Task. Álgebra Problem.

\title{
1 Introducción
}

El desarrollo profesional del docente se puede percibir a través de los cambios en su actuación en relación con diversos elementos que involucra su práctica, en palabras de Eraut (1977), implica un proceso natural de crecimiento profesional en que un profesor va adquiriendo, gradualmente, confianza, ganancias de nuevas perspectivas, incrementando en el conocimiento, descubriendo nuevos métodos y asumiendo nuevos roles.

En particular, los cambios en la actuación de los docentes pueden verse reflejados en relación con las tareas para el aula diseñadas por ellos para afrontar un su práctica. El diseño de la clase se aborda de una manera práctica delimitando qué se va hacer en la misma, formulando tareas que reflejan las expectativas de enseñanza y aprendizaje. Las tareas para el aula, se han estudiado profusamente en la literatura (DOYLE, 1983; PONTE, 2005; PONTE et al., 1997; RAMOS, 2011; STEIN et al., 2000) e incluso han sido objeto dentro de estudio de casos de la propia práctica profesional del investigador para comprender mejor ciertos aspectos de su trabajo, como lo menciona Ponte (2006).

En Chile, entre junio y octubre del año 2012, organizamos e implementamos un curso de formación continua, cuyo propósito fue promover la reflexión de los profesores respecto a problemas (que, desde ahora, llamaremos problemática) detectados por ellos en su práctica, en relación con la enseñanza y aprendizaje del álgebra con uso de tecnología. Luego que los profesores detectaran y profundizaran en sus problemáticas, en sesiones posteriores debían afrontarlas desde distintas perspectivas, incluyendo el diseño y análisis de tareas para el aula ${ }^{l}$, en diversas etapas de análisis e implementación. El objetivo de este documento es

\footnotetext{
${ }^{1}$ Para evitar ambigüedades, utilizaremos el término tarea para indicar las actividades de enseñanza que diseñan e implementan los profesores para lograr los objetivos de aprendizaje.
} 
describir la evolución que hemos apreciado en dos docentes que formulan e implementan una tarea durante su participación en este curso de formación.

\section{Antecedentes y marco de referencia}

Una variable frecuentemente empleada para analizar los cursos de formación de profesores son las tareas que los asistentes diseñan para el aula (CHVAL; LANNIN; BOWZER, 2008). Este tipo de análisis ha generado diversos marcos de referencia, como el marco de Chval, Lannin y Bowzer (2008), o la secuencia concéntrica de tareas para la enseñanza (VAN ZOEST; STOCKERO, 2008).

Doyle (1983) señala que una tarea se define considerando tres aspectos:

- Un producto o meta.

- Una serie de recursos disponibles en cada situación.

- Una serie de operaciones que se aplican sobre los recursos para conseguir dicha meta.

Stein y colaboradores (STEIN; SMITH, 1998; STEIN et al., 2000) destacan la demanda cognitiva puesta en juego en las tareas, distinguiendo tareas de nivel cognitivo bajo (memorísticas, procedimientos sin conexión) y alto (procedimientos con conexión y haciendo matemáticas). Este marco de tareas referente al nivel cognitivo puesto en juego en ellas, pone el énfasis en la actuación del profesor en clase, qué se materializa en las tareas que selecciona y la forma de gestionarlas, determinará maneras en que su instrucción apoya o inhibe la participación de los estudiantes en procesos de alto nivel cognitivo, y con ello en qué grado su actuación repercute en el aprendizaje del alumno. Una descripción de cada nivel de demanda cognitiva se ilustra en el Cuadro 1, sintetizada a partir del trabajo de Stein y colaboradores.

\begin{tabular}{|l|l|l|}
\hline $\begin{array}{l}\text { Nivel de } \\
\text { demanda } \\
\text { cognitiva }\end{array}$ & \multicolumn{1}{|c|}{ Tipo } & \multicolumn{1}{|c|}{ Descripción } \\
\hline $\begin{array}{l}\text { Baja } \\
\text { demanda } \\
\text { cognitiva }\end{array}$ & $\begin{array}{l}\text { Memorización } \\
\text { Procedimientos sin } \\
\text { conexiones }\end{array}$ & $\begin{array}{l}\text { Tareas para el aula automáticas, sólo se requiere la memoria, } \\
\text { sin realizar procedimientos. } \\
\text { Algoritmos que requieren uso de un procedimiento, pero no } \\
\text { demandan establecer conexiones entre conceptos } \\
\text { matemáticos. }\end{array}$ \\
\hline $\begin{array}{l}\text { Alta } \\
\text { demanda }\end{array}$ & $\begin{array}{l}\text { Procedimientos con } \\
\text { conexiones }\end{array}$ & $\begin{array}{l}\text { Involucran varios conceptos matemáticos subyacentes, con } \\
\text { múltiples representaciones, que ayudan a desarrollar el } \\
\text { significado de la tarea. No pueden ser resueltos } \\
\text { descuidadamente y su respuesta no se deduce explícitamente } \\
\text { del planteamiento. }\end{array}$ \\
\hline
\end{tabular}




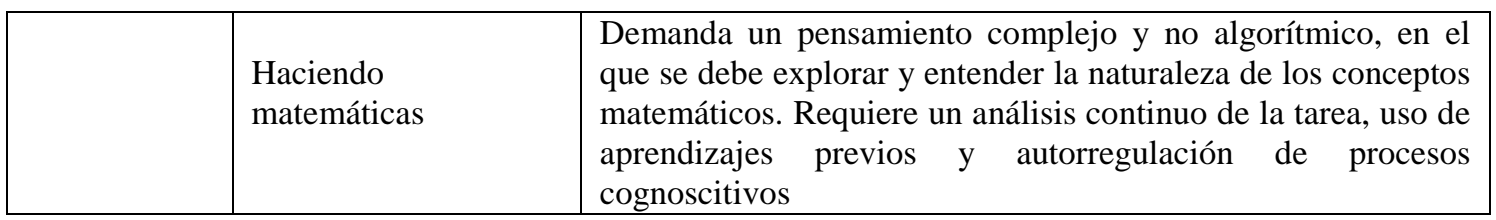

Cuadro 1- Niveles de demanda cognitiva de las tareas

Ponte (2005), concreta los componentes de Doyle, señalando que la tarea apunta a un determinado contenido matemático, es decir, a aspectos matemáticos implicados (hechos, conceptos, procedimientos, ideas) del currículo, buscando afrontar de manera sugerente los conceptos y procedimientos, proporcionando a los alumnos una buena oportunidad para implicarse en actividades matemáticas. A partir de estas apreciaciones, caracterizamos las tareas matemáticas para el aula a través de cuatro componentes: propósito o meta, recursos, demanda cognitiva y contenido matemático.

\section{Metodología}

Este trabajo, inmerso en una investigación más amplia que busca examinar la reflexión de los profesores sobre su práctica en el transcurso de un curso de formación continua, se sitúa en el paradigma cualitativo, de tipo descriptivo interpretativo (HERNÁNDEZ; FERNÁNDEZ; BAPTISTA, 2010), teniendo como instrumentos de recolección de información los portafolios y diarios de aprendizaje de cada docente, presentaciones de los profesores y transcripciones de vídeo de cada sesión. Realizamos un análisis de contenido, fijando como unidades referenciales, los conjunto de párrafos que tienen conexión o idea en común (KRIPPENDORFF, 1990).

El contexto del estudio se enmarca dentro de un curso para profesores de matemáticas chilenos, en el que distinguimos tres focos: identificar un problema (problemática) relativo al tratamiento del álgebra, llevando a cabo dos ciclos de un Estudio de Clases (ISODA; ARCAVI; MENA, 2007), es decir, diseño, realización y análisis de clases; y el empleo de la tecnología en la enseñanza.

Se realiza un estudio de caso, centrado en uno de los equipos de profesores participantes en el curso, compuesto por dos profesoras de enseñanza secundaria, quienes comenzaron planteándose una problemática sobre la modelación, pero posteriormente la concretaron en mitigar la dificultad que tienen los estudiantes para obtener la representación algebraica asociada a un problema de enunciado verbal. Se enfocaron en tareas para 
alumnos de 12 y 13 años, aproximadamente, y las profesoras decidieron emplear calculadoras gráficas TI Nspire junto a TI Navigator ${ }^{2}$.

Las categorías de análisis empleadas para el análisis de contenido de las producciones de las profesoras, surgen de la tipología de tareas presentadas, articuladas con el estudio que presentamos (Cuadro 2). En concreto, analizamos la categoría propósito a través de su relación con la problemática del grupo, es decir, en qué grado la las tareas abordan la problemática planteada por la pareja; examinamos el contenido matemático, en nuestro caso, el álgebra, analizando el rol que juega la variable en las tareas propuestas (FILLOY, 1999; KIERAN, 2007; TRIGUEROS et al., 1996) así como los sistemas de representación empleados por Gómez (2007), en donde añadimos una subcategoría diagramas, a partir de una de las lecturas (PUIG; CERDÁN, 1990) realizadas por el grupo para profundizar en la problemática; la categoría recursos, considera dos subcategorías, operaciones realizadas con dicho recurso en función de su uso educativo, y formato de trabajo o agrupamiento en función del recurso tecnológico (se trabajó de forma individual y grupal, con calculadoras para finalmente usar TI Navigator con un uso colectivo).

\begin{tabular}{|c|c|c|}
\hline Categorías & \multicolumn{2}{|r|}{ Subcategorías } \\
\hline Propósito & \multicolumn{2}{|c|}{$\begin{array}{l}\text { Aborda la problemática } \\
\text { Medianamente aborda la problemática } \\
\text { No aborda la problemática }\end{array}$} \\
\hline \multirow{4}{*}{ Demanda cognitiva } & \multirow[t]{2}{*}{ Baja } & Memorística \\
\hline & & Procedimiento sin conexión \\
\hline & \multirow[t]{2}{*}{ Alta } & Procedimiento con conexión \\
\hline & & Hacer matemática \\
\hline \multirow[t]{2}{*}{ Recursos } & $\begin{array}{l}\text { Operaciones } \\
\text { (uso educativo) }\end{array}$ & \begin{tabular}{l}
\multicolumn{4}{l}{$\begin{array}{l}\text { Verificador de resultados } \\
\text { Reforzador de ideas o } \\
\text { matemáticos }\end{array}$ conceptos } \\
$\begin{array}{l}\text { Construcción de ideas o } \\
\text { matemáticas }\end{array}$
\end{tabular} \\
\hline & $\begin{array}{l}\text { Formato de trabajo } \\
\text { o agrupamiento }\end{array}$ & $\begin{array}{l}\text { Individual } \\
\text { En grupos } \\
\text { Colectivo (clase completa) }\end{array}$ \\
\hline \multirow{5}{*}{ Contenido matemático } & \multirow[t]{2}{*}{ Representaciones } & $\begin{array}{l}\text { Gráficas } \\
\text { Tablas } \\
\text { Diagramas } \\
\text { Verbal }\end{array}$ \\
\hline & & $\begin{array}{l}\text { Algebraicas } \\
\text { Numéricas }\end{array}$ \\
\hline & \multirow[t]{3}{*}{ Rol de la variable } & Como incógnita \\
\hline & & Cómo número generalizado \\
\hline & & Cómo relación entre cantidades \\
\hline
\end{tabular}

Cuadro 2-Categorización de las tareas

\footnotetext{
${ }^{2}$ Programa que permite, visualizar el trabajo que cada alumno realiza en sus calculadoras, proyectándolo en una pantalla común.
} 
Los profesores diseñaron, implementaron y analizaron tareas, en diferentes momentos del proceso formativo. La figura 1 esquematiza el proceso de evolución de las tareas formuladas. $T_{i, j}$ corresponde a la tarea $i$, en el momento $j$ de su evolución. El curso comienza con la identificación y definición de la problemática y su ejemplificación con una tarea $\left(T_{1.0}\right)$. Dicha tarea evoluciona a través de nuevas formulaciones y nuevas tareas, y continúa en relación con los temas y momentos del curso, entre los que destacamos el momento de diseño de una clase, su realización y el análisis posterior. $T_{i, j}$ se diferencia de $T_{k, j}(i \neq k)$ cuando obedecen a diferentes contextos. En este artículo analizamos la evolución de la tarea $T_{1.0}$.

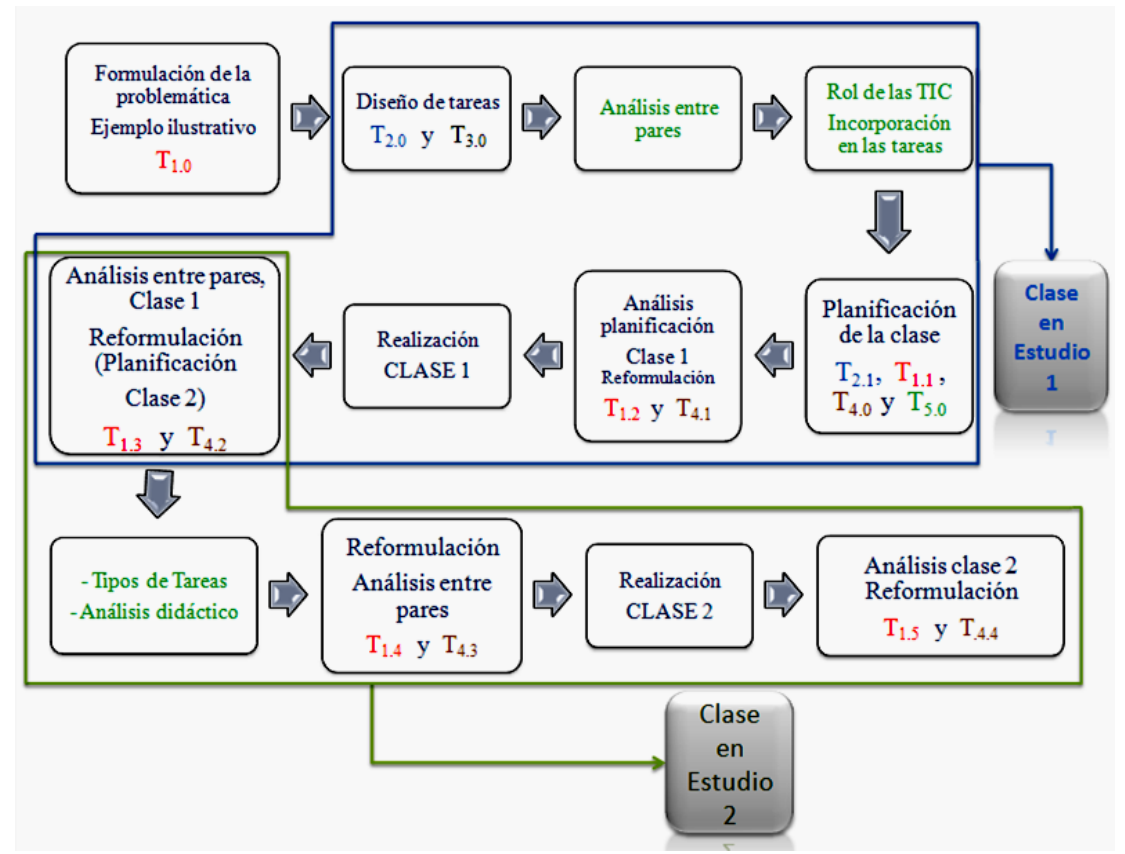

Figura 1-Presencia de las tareas en el curso de formación

\section{Análisis}

Al extraer de los instrumentos de recogida de datos las tareas propuestas por el grupo en cuestión, elaboramos el Anexo 1). En ella apreciamos tareas que el grupo ha detectado que requieren una mejora para utilizarlas, como la tarea $T_{1.0}$ (figura 2), manteniendo en ella el contexto y algunos elementos de su formulación. 


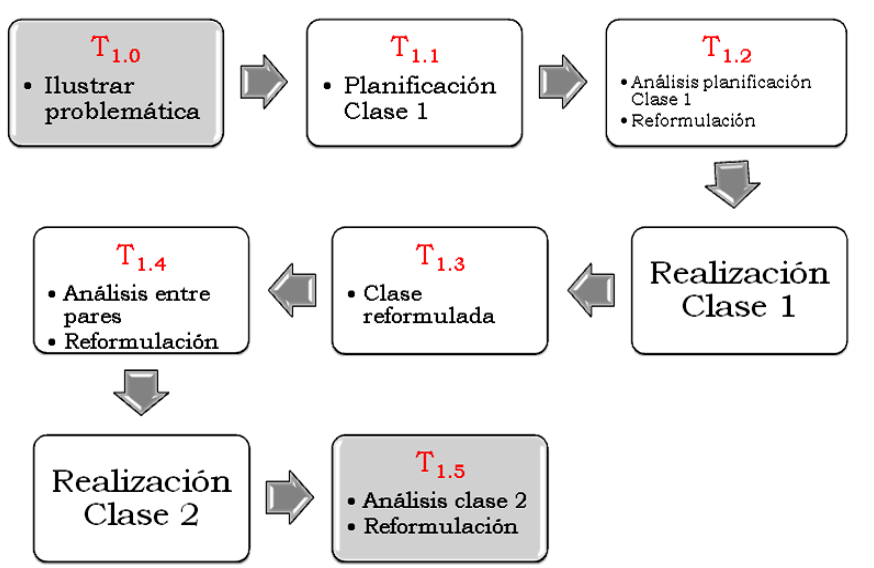

Figura 2-Transformaciones de la tarea $T_{1.0}$ dentro del curso de formación

Para este documento analizamos la evolución de esta tarea $\left(T_{1.0}\right)$, considerando aquellas versiones que provienen de una reformulación, dejando para un posterior análisis las que son implementadas o puestas en práctica, según indica Stein et al. (2000), pues éstas requieren de mayores elementos de análisis, como la comunicación en la clase.

En la tarea $T_{1.0}$, diseñada por las profesoras para ejemplificar su problemática, observamos que se trata de un problema que:

- Responde a las expectativas de un docente en términos instructivos, es decir, plantea una cuestión a los alumnos y dirige su trabajo a través de preguntas guiadas para lograr su propósito que se ajusta con la problemática planteada por el grupo. El enunciado plantea una pregunta, que se deja de lado en las preguntas posteriores.

- Se introduce en representación verbal, con intención de ir hacia la algebraica.

- Puede ser traducida por una ecuación de dos variables $(650 x+500 y=4400$, variable como incógnita). Demanda una expresión algebraica, que en este caso es una ecuación (igualdad), lo que más adelante se aprecia como un abuso de lenguaje, pues las docentes no parecen hacer distinción entre ecuaciones y expresiones algebraicas (variable como incógnita y como número generalizado).

- Requiere una identificación de elementos en una demanda cognitiva baja, en sus tres primeros apartados, con la idea de que los estudiantes llegarán a cubrir el cuarto apartado del enunciado de la tarea (de demanda cognitiva más elevada, requiere establecer conexiones entre datos y variables).

- Por el momento en que se sitúa en el curso de formación, no incluye uso de tecnología.

De manera similar, hemos analizado las cinco tareas que la siguen, $T_{1.1}, T_{1.2}, T_{1.3}, T_{1.4} \mathrm{y}$ $T_{1.5}$. 
Para profundizar en la problemática, las profesoras realizan algunas lecturas sobre la transición de la aritmética al álgebra y sobre el rol de la variable en álgebra (FILLOY, 1999; TRIGUEROS et al., 1996), y concluyen diseñando tareas para una clase. Presentan estas tareas a sus pares y el grupo las reformulan. En esta etapa del curso, se aborda el papel de la tecnología en la enseñanza, y se les impone modificar las tareas incorporando tecnología. Eso da origen a la tarea $T_{1.1}$ en la que apreciamos cambios (Cuadro 3).

Se discute en el curso esta tarea propuesta para clase y se reformula posteriormente, dando lugar a $T_{1.2}$, prevista para la clase 1. Así, finaliza la etapa de planificación de la clase empleando tecnología. Posteriormente, una de las profesoras implementa la clase en uno de sus cursos. Luego del análisis de ésta junto a sus pares y su consecuente reformulación, surge la tarea $T_{1.3}$. Esta tarea está inmersa dentro de la planificación que cierra el primer ciclo de Estudio de Clases (la reformulación de la clase 1), pero, a la vez, es la que inicia el segundo ciclo de una Clase en Estudio (planificación clase 2).

\begin{tabular}{|c|c|c|}
\hline Categoría & Cambios & Explicitación \\
\hline \multirow[t]{3}{*}{$\begin{array}{l}\text { Contenido } \\
\text { matemático }\end{array}$} & $\begin{array}{l}\text { Se mantiene el tópico matemático } \\
\text { involucrado }\end{array}$ & \\
\hline & $\begin{array}{l}\text { Introduce una tercera representación, la } \\
\text { gráfica }\end{array}$ & Con intención de incorporar tecnología \\
\hline & $\begin{array}{l}\text { Se precisa, demandando ecuación, no } \\
\text { expresión algebraica (rol de la variable) }\end{array}$ & $\begin{array}{l}\text { La discusión entre pares sobre la tarea } \\
\text { anterior cuestiona el término expresión } \\
\text { algebraica y precisa diferencia con } \\
\text { ecuación }\end{array}$ \\
\hline Propósito & $\begin{array}{l}\text { El propósito sigue siendo la } \\
\text { representación algebraica, y para facilitar } \\
\text { la construcción de los alumnos, sugiere } \\
\text { que contrasten las representaciones en } \\
\text { forma de rectas que construye la } \\
\text { calculadora. (Consideramos que es } \\
\text { medianamente relativo a la } \\
\text { problemática) }\end{array}$ & $\begin{array}{l}\text { Observaciones significativas }{ }^{3} \text { sobre la } \\
\text { exposición a sus pares de las tareas } \\
\text { propuestas: } \\
\text { Las preguntas son muy conductistas, } \\
\text { entonces: utilizar las estrategias de los } \\
\text { alumnos para modelar. Se puede apoyar } \\
\text { con preguntas a modo de ir incluyendo }\end{array}$ \\
\hline \multirow[t]{2}{*}{$\begin{array}{l}\text { Recurso } \\
\text { (Calculadora) }\end{array}$} & Se usa como verificador de respuestas & $\begin{array}{l}\text { Aprecian cubrir dos objetivos: incorporar } \\
\text { tecnología y darle papel verificador. }\end{array}$ \\
\hline & $\begin{array}{l}\text { Se especifica trabajo colectivo para } \\
\text { contraste (forma de trabajo o } \\
\text { agrupamiento) }\end{array}$ & \\
\hline $\begin{array}{l}\text { Demanda } \\
\text { cognitiva }\end{array}$ & Se mantiene nivel cognitivo anterior & $\begin{array}{l}\text { Ambas tareas requieren uso de un } \\
\text { procedimiento, pero no demandan } \\
\text { establecer conexiones entre conceptos } \\
\text { matemáticos. }\end{array}$ \\
\hline
\end{tabular}

Cuadro 3- Análisis de transformaciones de la tarea $T_{1.0}$ a $T_{1.1}$

Se incorporan nuevos elementos teóricos en el curso de formación que puedan apoyar el diseño y análisis de las tareas, en particular se hace la diferencia entre ejercicio y problema, se estudia la demanda cognitiva asociada a una tarea y se desarrollan, con mayor detalle, los

\footnotetext{
${ }^{3}$ Dentro del portafolio las docentes colocan aspectos mencionados por sus pares que consideran significativos para ellas, a estos comentarios llamaremos observaciones significativas.
} 
elementos más relevantes del análisis didáctico (GÓMEZ, 2007) del contenido matemático involucrado en cada problemática. A partir de esto, los grupos vuelven a replantearse su planificación lo que lleva a las profesoras a formular la tarea $T_{1.4}$, que se pretende llevar al aula. Luego la clase es analizada en el curso de formación surgiendo la versión reformulada, en donde se enmarca la tarea $T_{1.5}$.

Al llevar a cabo un análisis exhaustivo por tarea (que por razones de espacio no se detallarán en este documento) hemos observado cambios en sus formulaciones, de los que queremos mostrar cómo el contenido matemático se ve influenciado por el recurso tecnológico que se incorporan, provocando que las profesoras reconsideren en dos ocasiones la función involucrada por la dificultad en la representación gráfica que se realiza con la calculadora (Cuadro 4).

\begin{tabular}{|c|c|c|}
\hline \multicolumn{2}{|c|}{ Tópico involucrado } & $\begin{array}{l}\text { Representaciones (además de la } \\
\text { verbal y algebraica) }\end{array}$ \\
\hline$T_{1.0}$ & $\begin{array}{l}\text { Involucra una función de dos variables (variable como relación } \\
\qquad f: \mathfrak{R} x \mathfrak{R} \rightarrow \mathfrak{R} \\
\text { entre cantidades) de la forma }(x, y) \mapsto 650 x+500 y \\
\text { aún no conocen los alumnos del nivel escolar }(13 \text { años } \\
\text { aproximadamente) donde se aplica. Pero, es posible tratarla como } \\
\text { una ecuación de dos variables }(650 x+500 y=4400 \text {, variable como } \\
\text { incógnita), que es como las profesoras la abordan. }\end{array}$ & --------- \\
\hline$T_{1.1}$ & Se mantiene contenido & Gráfica (como verificadora) \\
\hline$T_{1.2}$ & $\begin{array}{l}\text { Se cambia contenido matemático, a una función discreta de la forma } \\
y=40 x \text { con } x \in \aleph, \text { aunque está presente (en la } \\
\text { representación gráfica) la función continua de dominio real asociada } \\
\text { a ella }\end{array}$ & $\begin{array}{l}\text { Gráfica, diagrama y tablas } \\
\text { (verificadoras de la expresión } \\
\text { algebraica) }\end{array}$ \\
\hline$T_{1.3}$ & $\begin{array}{l}\text { Cambian la función discreta de la tarea anterior por una función } \\
\text { continua de la forma } y=850 x \text {, la que se corresponde con la } \\
\text { representación gráfica que utilizan en la calculadora. }\end{array}$ & $\begin{array}{l}\text { Se elimina la representación } \\
\text { gráfica y de tablas } \\
\text { Continúa uso de diagramas } \\
\text { (verificadora) }\end{array}$ \\
\hline$T_{1.4}$ & Se mantiene contenido & $\begin{array}{lllr}\text { Diagramas } & \text { (para } & \text { apoyar } & \text { la } \\
\text { transición } & \text { entre } & \text { verbal } & y \\
\text { algebraico) } & & & \\
\end{array}$ \\
\hline$T_{1.5}$ & Se mantiene contenido & \\
\hline
\end{tabular}

Cuadro 4- Contenido matemático y representaciones de las tareas analizadas

Otro aspecto que se aprecia en el Cuadro 4, está relacionado con los sistemas de representación empleados, ya que con intención de incorporar la tecnología se agrega la representación gráfica en la tarea $T_{1.1}$ y $T_{1.2}$, pero con un rol verificador de respuestas de los alumnos. A raíz de las discusiones entre pares se elimina esta representación y se da mayor protagonismo al uso de diagramas, tomando finalmente un rol mediador en la transición de la representación verbal a la algebraica. Esto refleja además una atención de las profesoras al 
concepto de modelación que está detrás de su problemática, como queda de manifiesto en la siguiente observación escrita en el diario de aprendizaje de una de las profesoras del grupo:

Se puede utilizar un método más gráfico para ir trabajando en paralelo a la modelación, en vez de que sea con las preguntas que son muy conductistas... esta nueva versión considera partir del diagrama para luego, a partir de él, encontrar la representación algebraica...

\section{Comentarios finales}

La selección de las tareas para el aula es uno de los aspectos relevantes dentro de las funciones diarias que realiza un profesor (PONTE, 2005), por ello es esencial tener presente los elementos relevantes que permitan analizarlas. Nuestra caracterización obedece a diversos componentes apreciables a la hora de diseñar, realizar y discutir una tarea para el aula.

Operativizar esta caracterización de las tareas propuestas por el grupo del curso de formación, en término de objetivos, contenido, recursos y demanda cognitiva nos permitió ver las transformaciones que éstas han sufrido a lo largo del proceso formativo, destacamos:

- Una evolución en la atención de las profesoras al rol de la variable. Las primeras tareas manifiestan mezcla de roles, lo que se elimina a partir de la tarea $T_{1.5}$. También reconsideran el uso de una función discreta (variable como relación funcional discreta) sobre una función lineal (variable como una relación funcional continua).

- Una toma de conciencia en la adecuada elección de las representaciones óptimas para el desarrollo de la tarea: desde el empleo de gráficas al uso de diagramas como apoyo a la construcción de la representación algebraica.

- Mejor atención al propósito en el que se enmarca la tarea, lo que refleja mayor profundización en la problemática del grupo.

- Preocupación por aumentar la demanda cognitiva, dando importancia a las conexiones entre las representaciones requeridas en la actividad y en que estén las instrucciones explícitas (en el enunciado), empleando diagramas para percibir los pasos para la construcción de la representación algebraica.

En paralelo con esta variación en la formulación, se aprecia que las profesoras contemplan la problemática con otros ojos, por ejemplo, en relación con el significado que le atribuyen a la modelación, disponiendo de una formulación más precisa, lo que se manifiesta en apreciaciones como: "El modelamiento es un proceso largo. Dentro de los pasos que lleva 
el modelamiento son identificar la situación, organizar los datos, representarlos, buscar estrategias [...]" (extracto de uno de los portafolios del grupo).

En la evolución de la tarea planteada apreciamos que las docentes incorporan nuevos elementos de actuación que aparece en las tareas para el aula, ganando en nuevas perspectivas sobre el diseño de éstas, incrementando el conocimiento específico frente a la noción de modelación, pero además, descubriendo nuevos métodos como el propuesto por Puig y Cerdán (1990). Estos elementos nos ayudan a mostrar cómo las profesoras afrontan un problema de su práctica y avanzan en su desarrollo profesional.

A raíz de este estudio, dejamos abiertas nuevas líneas de trabajo, como profundizar en la caracterización de las tareas para el aula, contemplando otros elementos que permitan analizarlas desde otras perspectivas. Ésta y otras líneas puedan surgir de esta lectura, cuyas orientaciones puedan ayudarnos a avanzar en el estudio del desarrollo profesional del docente de matemática a la luz de las tareas para el aula propuestas por ellos.

\section{Referencias}

CHVAL, K.; LANNIN, J.; BOWZER, A.The Task Design Framework: Considering Multiple Perspectives in an Effective Learning Environment for Elementary Preservice Teachers. In: ARBAUG, F.; TAYLOR, P.M. (Ed.) Inquiry into Mathematics Teacher Education. San Diego: AMTE, 2008. p. 35-45.

DOYLE, W. Academic work. Review of Educational Research. Washington, DC, v. 53, n. 2, p.159199, summer. 1983.

ERAUT, M. Strategies for Promoting Teacher Development. British Journal of in service Education, v.4, n.12, 95-99. 1977.

FILLOY, E. Aspectos teóricos del álgebra educativa. México D.F.: Grupo Editorial Iberoamérica, 1999.

GÓMEZ, P. Desarrollo del conocimiento didáctico en un plan de formación inicial de profesores de matemáticas de secundaria. 2007. 482f. Tesis (Doctoral en Didáctica de las Matemáticas) Departamento de Didáctica de la Matemática, Universidad de Granada, Granada, 2007. HERNÁNDEZ, R., FERNÁNDEZ C.; BAPTISTA, P. Metodología de la investigación. México: McGraw-Hill, 2010.

ISODA, A.; ARCAVI, A.; MENA A. EI Estudio de Clases Japonés en Matemáticas. Su importancia para el mejoramiento de los aprendizajes en el escenario global. Valparaíso: Ediciones Universitarias de Valparaíso, 2007.

KIERAN, C. Learning and Teaching Algebra at the Middle School Through College Levels. In: LESTER, F. K. (Ed.). Second Handbook of Research on Mathematics Teaching and Learning. Reston, Virginia: NCTM e IAP. 2007. p.707-762. 
KRIPPENDORFF, K. Metodología de análisis de contenido: teoría y práctica. Barcelona: Paidós, 1990.

PONTE, J. P. Gestão curricular em Matemática. In: GTI (Ed.) O professor e o desenvolvimento curricular.Lisboa: APM. 2005. p. 11-34.

PONTE, J. P. Estudos de Caso em Educação Matemática. BOLEMA-Boletim de Educação

Matemática, Rio Claro, SP, v. 19, n. 25, 2006, p. 105-132.

PONTE, J. P; BOAVIDA, A.; GRAÇA, M.; ABRANTES, P. Didáctica da Matemática. Lisboa: DES do ME, 1997, p. 134.

PUIG, L.; CERDÁN, F. Acerca del carácter aritmético o algebraico de los problemas verbales. En: FILLOY, E.; ROJANO, T. (Eds.) Memorias del Segundo Simposio Internacional sobre Investigación en Educación Matemática. Cuernavaca, Morelos: PNFAPM. 1990. p. 35-48.

RAMOS, E. Reflexión de docentes sobre la enseñanza del álgebra en un programa formativo. 2001. 209f. Disertación (Máster en Didáctica de la Matemática)- Departamento de Didáctica de las Matemáticas, Universidad de Granada, Granada, 2011.

STEIN, M. K.; SMITH, M. S. Mathematical tasks as a framework for reflection: from research to practice. Mathematics Teaching in the Middle School, Reston, VA, EUA, v. 3, n.4, p. 268 - 275. Enero.1998.

STEIN, M. K.; SMITH, M. S.; HENNINGSEN, M. A.; SILVER, E. A. Implementing standardsbased mathematics instruction: a casebook for professional development, Reston: NCTM, 2000.

TRIGUEROS, M.; REYES, A.; URSINI, S.; QUINTERO, R. Diseño de un cuestionario de diagnóstico acerca del manejo del concepto de variable en el álgebra. Enseñanza de las Ciencias, Barcelona, v. 14, n. 3, p.351-363, junio, 1996.

VAN ZOEST, L.; STOCKERO, S. Concentric Task Sequences: A Model for Advancing Instruction Based on Student Thinking. In: Arbaugh, F.; Taylor, P. M. (Ed.) Inquiry into Mathematics Teacher Education, San Diego, CA: AMTE. 2008. p. 47-58.

Submetido em Dezembro de 2013. Aprovado em Julho de 2014. 


\section{Anexo 1}

\begin{tabular}{|c|c|}
\hline $\begin{array}{l}\text { Códigota } \\
\text { rea }\end{array}$ & Tarea \\
\hline $\mathrm{T}_{1.0}$ & $\begin{array}{l}{ }^{4} \text { Lee el siguiente problema y responde: Tomás compró en la feria naranjas y manzanas y } \\
\text { gastó } \$ 4400 . \text { El kilo de naranjas le costó } \$ 650 \text { y el de manzanas } \$ 500 \text { ¿Cuántos kilos compró } \\
\text { Tomás de cada cosa? } \\
\text { a) ¿cuántas variables se pueden apreciar en el problema? } \\
\text { b) ¿cuáles son? ¿de qué manera las representarías? } \\
\text { c) ¿qué otros datos se representan en el problema? } \\
\text { d) ¿qué expresión algebraica puede representar el problema? }\end{array}$ \\
\hline $\mathrm{T}_{1.1}$ & $\begin{array}{l}\text { Se presenta un problema, el cual los estudiantes deben representar algebraicamente en la } \\
\text { calculadora. } \\
\text { Tomás compró mandarinas y pimentones, y gastó } \$ 4400 \text {. El kilo de mandarinas le costó } \$ 650 \\
\text { y el de pimentones } \$ 500 \text {. } \\
\text { ¿Cuál es la ecuación que permite calcular esta ecuación? } \\
\text { Se comentan respuestas de los estudiantes mediante el software. } \\
\text { El profesor presenta (con TI Navigator) una gráfica que muestra las soluciones del problema. } \\
\text { Los estudiantes introducen en las calculadoras la ecuación descrita por ellos, verificando si su } \\
\text { ecuación presenta las mismas soluciones expuestas por el profesor. } \\
\text { Se discuten las soluciones obtenidas por los estudiantes, analizando los posibles errores. }\end{array}$ \\
\hline $\mathrm{T}_{1.2}$ & $\begin{array}{l}\text { Se presenta el siguiente problema, escribiéndolo en el pizarrón, el cual puede ser acompañado } \\
\text { de un dibujo que lo haga más concreto. } \\
\text { En el almacén de don Luis se venden masticables a } \$ 40 \text { cada uno } \\
\text { Escribe una expresión algebraica qué represente esta situación. } \\
\text { En parejas se comparte la expresión descrita y lo escriben en la calculadora (utilizan distintos } \\
\text { símbolos). } \\
\text { Para comparar las expresiones se utiliza la gráfica de la función utilizada, los estudiantes } \\
\text { introducen su expresión. } \\
\text { Como utilizan símbolos que la calculadora no reconoce, se definen las variables utilizando las } \\
\text { letras y, x. Con ello, se explica que esto describe una función, donde la x es una variable } \\
\text { independiente y la y es la variable dependiente. } \\
\text { Vuelven a introducir la expresión, ahora utilizando los nuevos símbolos, se proyectan las } \\
\text { gráficas que representan las soluciones y se busca la concordancia entre ellas, analizando } \\
\text { todas aquellas que no llegaron a las soluciones, escribiéndolas a un lado del pizarrón. } \\
\text { Se vuelve al problema escrito en la pizarra, se pide a los estudiantes leerlo e identificar } \\
\text { primero las variables involucradas, a ellas se les asigna un símbolo, luego se describen } \\
\text { situaciones para el problema, buscando identificar la operación que la representa (se puede } \\
\text { realizar una tabla de doble entrada para apoyarse). } \\
\text { Se construye un diagrama que represente el problema en el pizarrón y se concluye escribiendo } \\
\text { la función nuevamente y realizando la gráfica. }\end{array}$ \\
\hline $\mathrm{T}_{1.3}$ & $\begin{array}{l}\text { Moisés compra, en el almacén de Don Luis, pan a } \$ 850 \text { el kilogramo. Representen } \\
\text { algebraicamente esta situación. } \\
\text { Construyen el diagrama que representa la situación anterior, a partir de las herramientas de la } \\
\text { calculadora TI. }\end{array}$ \\
\hline $\mathrm{T}_{1.4}$ & $\begin{array}{l}\text { Se comparte con los estudiantes el objetivo de la clase, motivándoles a atender la importancia } \\
\text { de éste y su significado } \\
\text { Se les hace entrega de una hoja con la siguiente situación multicopiada, la cual puede ser } \\
\text { acompañada de un dibujo que lo haga más concreto. (Se pide a los estudiantes que utilicen } \\
\text { lápiz pasta para escribir las ecuaciones en las hojas y que no las borren). } \\
\text { Moisés compra, en el almacén de Don Luis, pan a } \$ 850 \text { el kilogramo. ¿Cuál (es) la } \\
\text { representación algebraica que podría asociarse a la situación? } \\
\text { Explica, justificando los pasos que utilizaste para llegar a dicha representación(es). } \\
\text { En grupos ingresarán la solución a la calculadora TI, destacando los pasos que utilizaron para } \\
\text { representarla. } \\
\text { Para comparar las ecuaciones se proyectan las soluciones, un integrante de cada grupo debe } \\
\text { explicar su solución y los pasos que utilizaron para llegar a dicha representación. }\end{array}$ \\
\hline
\end{tabular}

${ }^{4}$ Destacamos en cursiva el enunciado de las tareas. 


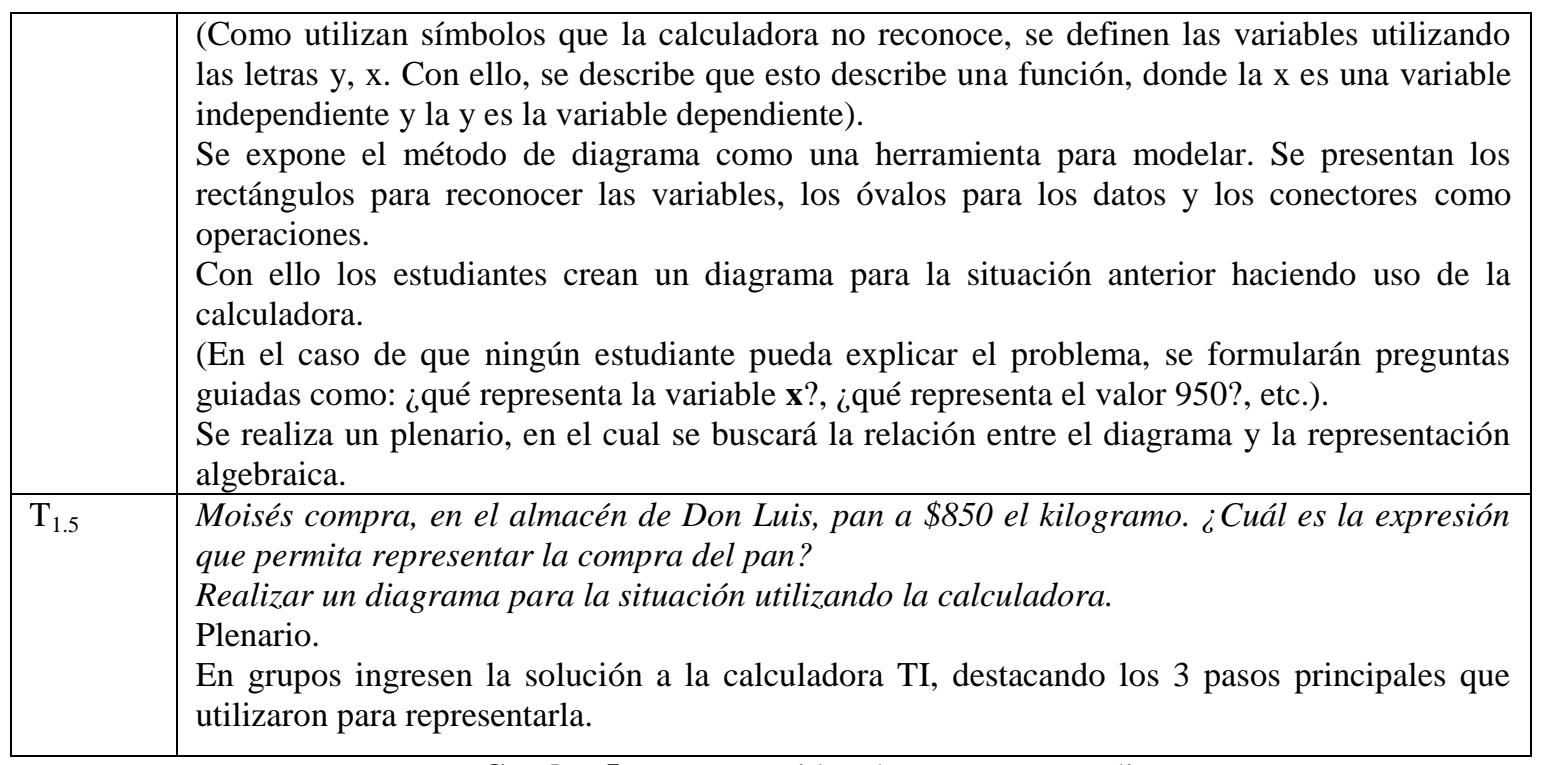

Cuadro 5-Tareas consideradas para este estudio 